динитрофенил) гидразон-1,3,5-триазинан-2,4диона // Universum: Химия и биология : электрон. научн. журн. 2020. № 6(72). URL: http://7universum.com/ru/nature/archive/item/9514

10. Пучков Сергей Вениаминович. Компьютерные технологии в науке, технике, образовании. Расчеты физико-химических и термодинамических характеристик органических соединений: методические указания к лабораторным работам и самостоятельной работе. - Кемерово: КузГТУ, 2013. - С. 23

11. Краткий справочник физико-химических величин / под ред. А. А. Равделя, А. М. Пономарёвой. - 9-е изд., перераб. и доп. - СПб.: Специальная Литература, 1999. - С. 232

12. Ганиев Б.Ш., Холикова Г.К., Салимов Ф.Г. Использование циануровой кислоты в качестве дезинфицирующих средств для окружающей среды. Материалы международной научной конференции «Инновационные решения инженерно-технологических проблем современного производства». 2 ТОМ. 14-16 ноябр. Бухара, -2019. С. 21-23

13. ChemOffice // CambridgeSoft [Electronic resource]. - Mode of access : http://www.cambridgesoft.com/software/details/?ds= 1. - Date of access : 22.11.2010.
14. Соловьев, М.Е. Компьютерная химия / М. Е. Соловьев, $\mathrm{M}$. Соловьев. - М. : Солон-Пресс, 2005. - 536 с.

15. Литвак М.M. Расчет геометрических параметров и конформационный анализ 1,2-Оцианэтилиденовых производных углеводов методом молекулярной механики // Научные ведомости Белгородского государственного университета. Серия: Естественные науки. -2012. T.21. - №.21 (140)

16. Литвак М.М. Компьютер как инструмент исследования при изучении химии и смежных дисциплин // Научные ведомости Белгородского государственного университета. Серия: Гумантарные науки. -2014. - Т.21. - №.6 (177)

17. Shibayama, A.; Kajiki, R.; Kobayashi, M.; Mitsunari, T.; Nagamatsu, A. 6-Acyl-1,2,4-triazine3,5-dione derivative and herbicides. Patent WO 2012002096; Chem. Abstr. 2012, 156, 122559.

18. Fesenko, Anastasia A., et al. "N2-Alkylation of semicarbazones. A general and efficient protocol for the synthesis of 2-alkylsemicarbazides from semicarbazide." Organic Chemistry part ii (2019): 176189.

19. Абдурахмонов С. Ф. и др. Синтез и исследование биядерных комплексов ванадила(II) на основе бис-5-оксипиразолинов //Universum: химия и биология. - 2019. - №. 12 (66). -С. 50-55

УДК: [546.56 + 546.19]:546-54.05

СОЛВОТЕРМАЛЬНЫЙ СИНТЕЗ ТРОЙНЫХ НАНОСОЕДИНЕНИЙ ВІ̇ВВЕ

Гараев А.М., Рзаева А.Б. Нахчьванское Отделение НАН Азербайджана, Институт Природных Ресурсов

\title{
SOLVOTHERMAL SYNTHESIS OF TRIPLE NANO COMPOUNDS BISBSE 3 IN A LIQUID PHASE
}

\author{
A.M. Garayev, A. B. Rzayeva \\ Nakhchivan Branch of Azerbaijan NAS, \\ Institute of Natural Resources
}

АННОТАЦИЯ

DOI: 10.31618/ESU.2413-9335.2020.5.76.931

Тройные наносоединение висмут сурьмы селенида синтезированы в сольвотермальных условиях в этиленгликолевой среде в интервале температур 453-463 К в течение 15 часов из оксида висмута(III), оксида сурьмы(III), элементарный селена (аморф) и гидразин моногидрата. При температуре 453-463 К после 15 часового синтеза получается хлопьевидный осадок. Выполнены термографический, дифференциально-термические (ДТА), рентгеногра-фический (РФА), химический, и морфологический анализы соединение и установлено, что кристаллы соединения представлены в виде нано и микрополочки. Результаты показали, что состав селенида висмута сурьмы соответствует формулам $\mathrm{BiSbSe}_{3}$

\section{ABSTRACT}

Ternary compounds of bismuth antimony selenide have been synthesized under solvothermal conditions in ethylene glycol medium at the temperature of 453-463 K during 15 hours from bismuth (III) oxide, antimony (III) oxide, elemental selenium (amorph) and hydrazine monohydrate. At a temperature of 453-463 K, after a 15-hour synthesis, a flocculent precipitate is obtained. The thermographic, differential thermal (DTA), X-ray (XRD), chemical, and morphological analyses of the compound were performed and it was found that the crystals of the compound are presented in the form of nano and micro-shelves. The results showed that the composition of selenium compounds of antimony bismuth corresponds to the BiSbSe 3 formulas

Ключевые слова: солвотермальный метод, висмут сурьмы селенида этиленгликолевой среде, наночастицы, ДТА, РФА, нано, микрополочки.

Keywords: solvothermal, bismuth (III) oxide, antimony (III) oxide antimony bismuth selenide, ethylene glycol medium, nanoparticles, DTA, XRD, nano, micro-shells. 


\section{ВВЕДЕНИЕ}

В связи с растущим глобальным энергетическим кризисом, опасным воздействием на окружающую среду и ограниченным запасом ископаемого топлива, потребность в каком-либо альтернативном источнике энергии является значительной. Термоэлектрические материалы пришли, чтобы спасти мир от растущего энергетического кризиса. Отработанное тепло, исходящее от автомобилей и других электроприборов, можно эффективно использовать, вырабатывая из него электричество, не добавляя больше загрязнения в атмосферу, что возможно только с помощью термоэлектрических материалов. Термоэлектрические материалы имеют широкий спектр применения: от мелкомасштабного производства электроэнергии до использования в ракетах и космических кораблях.

Бинарные и тройные халькогениды, такие как теллурид висмута, селенид висмута, теллурид сурьмы, селенид сурьмы, висмут селенид сурьмы, висмут теллурид сурьмы и их сплавы, имеют репутацию эффективных термоэлектрических материалов при комнатной температуре и широко используются для термоэлектрического охлаждения. В умеренном диапазоне температур источника тепла эти материалы также могут быть использованы в термоэлектрических генераторах.

Тройные халькогенидные стекла $\mathrm{BiSbSe}_{3}$ были синтезированы с помощью обычной технологии прямого синтеза. Рентгеновская дифракция, просвечивающая электронная микроскопия и сканирующая электронная микроскопия были использованы для определения структурных и морфологических характеристик[1] Поликристаллические сплавы $\mathrm{Bi}_{48-\mathrm{x}} \mathrm{Sb}_{\mathrm{x}} \mathrm{Se}_{52}$ (где х= $0.6,25,35$ и 44 ат.\%) были получены методом прямой монотемпературы. Морфологию поверхности, кристаллическую природу и композиционный анализ изучали с помощью сканирующей электронной микроскопии, рентгеновской дифракции. (XRD) и энергодисперсионная рентгеновская спектроскопия (EDX) соответственно. Оптические измерения показали, что сплавы $\mathrm{Bi}_{48}-$ х $\mathrm{Sb}_{\mathrm{x}} \mathrm{Se}_{52}$ являются полупроводниками с прямой запрещенной зоной. Было обнаружено, что энергия активации и оптическая запрещенная зона увеличиваются с ростом увеличение концентрации

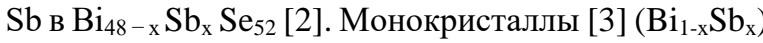
$2 \mathrm{Se}_{3}$ ( $\mathrm{x}=0$ до 0.2 ), полученные из элементов с помощью модифицированного метода Бриджмена, характеризовались измерениями инфракрасного отражения и коэффициента пропускания. Интересно, [4] что $\mathrm{BiSbSe}_{3}$, легирующий продукт с $50 \% \mathrm{Sb}$ в числах $\mathrm{Bi}$, демонстрирует выдающиеся переносы электронов и фононов. $\mathrm{BiSbSe}_{3}$ имеет ромбическую структуру и имеет много проводящих зон, которые одновременно увеличивают электропроводность и коэффициенты Зеебека. Полученные результаты показывают, что $\mathrm{BiSbSe}_{3}$ является новым и надежным кандидатом для производства ТЕ энергии в диапазоне средних температур. Для улучшения термоэлектрических характеристик $\mathrm{BiSbSe}_{3}$ n-типа было осуществлено кодирование и анионное кодирование путем легирования $\mathrm{SnCl}_{4}$. Легирование $\mathrm{SnCl}_{4}$ в основном регулирует взаимосвязь между электропроводностью и коэффициентом Зеебека, что является эффективным способом повышения добротности ZT[5].

Анализ литературного материала показывает, что $\mathrm{BiSbSe}_{3}$ были получены только методом ампульного синтеза из элементарных веществ. В литературе отсутствуют материалы по получению нано- и микрочастиц. В представленной работе исследовано влияние условий осаждения аморфнокристаллических нано- и микрочастиц $\mathrm{BiSbSe}_{3}$ из этиленгликолевых растворов.

\section{МЕТОДИКА ЭКСПЕРИМЕНТА}

Тройные халькогенидные соединения $\mathrm{BiSbSe}_{3}$ были синтезированы из оксида висмута(III), оксида сурьмы(III), элементарный селена (аморф) и гидразин моногидрата в солвотермальных условиях. Сначала оксида висмута(III), оксида сурьмы(III), элементарный селена (аморф) соответствие стехиометрией $\mathrm{BiSbSe}_{3}$, смешивается в 20 мл этиленгликоле, затем добавляется как восстановительный реагент гидразин моногидрата $(\mathrm{pH}=10-11)$. Смесь нагревается в герметичной стеклянной ампуле, помещенной в микроволновую электрическую печь (Speedwave four BERGHOF Германия). Проба в течение 15 часов при температурном диапазоне 453 до 463 К сохраняется в печи. Полученный осадок фильтруется через стеклянный фильтр, промывается разбавленным раствором соляной кислоты, ультрачистой водой, наконец, этиловым спиртом, высушивается при 333-343 К в вакууме. Уровнение реакции можно написат так:

$$
\mathrm{Bi}^{+3}+\mathrm{Sb}^{+3}+3 \mathrm{Se}^{-2}=\mathrm{BiSbSe}_{3}
$$

Состав полученного соединения (соотношение)Bi:Sb:Se определен в дериватографе NETZSCH STA 449F349F3 (Германия) и химическим анализом. Дифференциальнотермический анализ (ДТА) проведен пирометром HTP-71, Thermoscan-2. Фазовый анализ BiSbSe 3 изучен с помощью рентгеновым дифрактометром D8 ADVANCE "Bruker" (CuK $\alpha, \lambda=1.5406 \AA, 0<2 \theta$ $<80^{\circ}$ ). Морфологические исследования были выполнены с помощью сканирующей электронной микроскопии ТМ3000 (Hitachi, Япония).

\section{ОБСУЖДЕНИЕ РЕЗУЛЬТАТОВ}

Известно, что в зависимости от условия получения халькогениды в органической или водной среде, синтезируются по состав может быть с различной стехиометрии. Поэтому, синтезируемых солвотермальным методом $\mathrm{BiSbSe}_{3}$ проведены термогравиметрический анализы в приборе NETZSCH STA 449F349F3. Результаты анализов проведены на рисунке 1. 


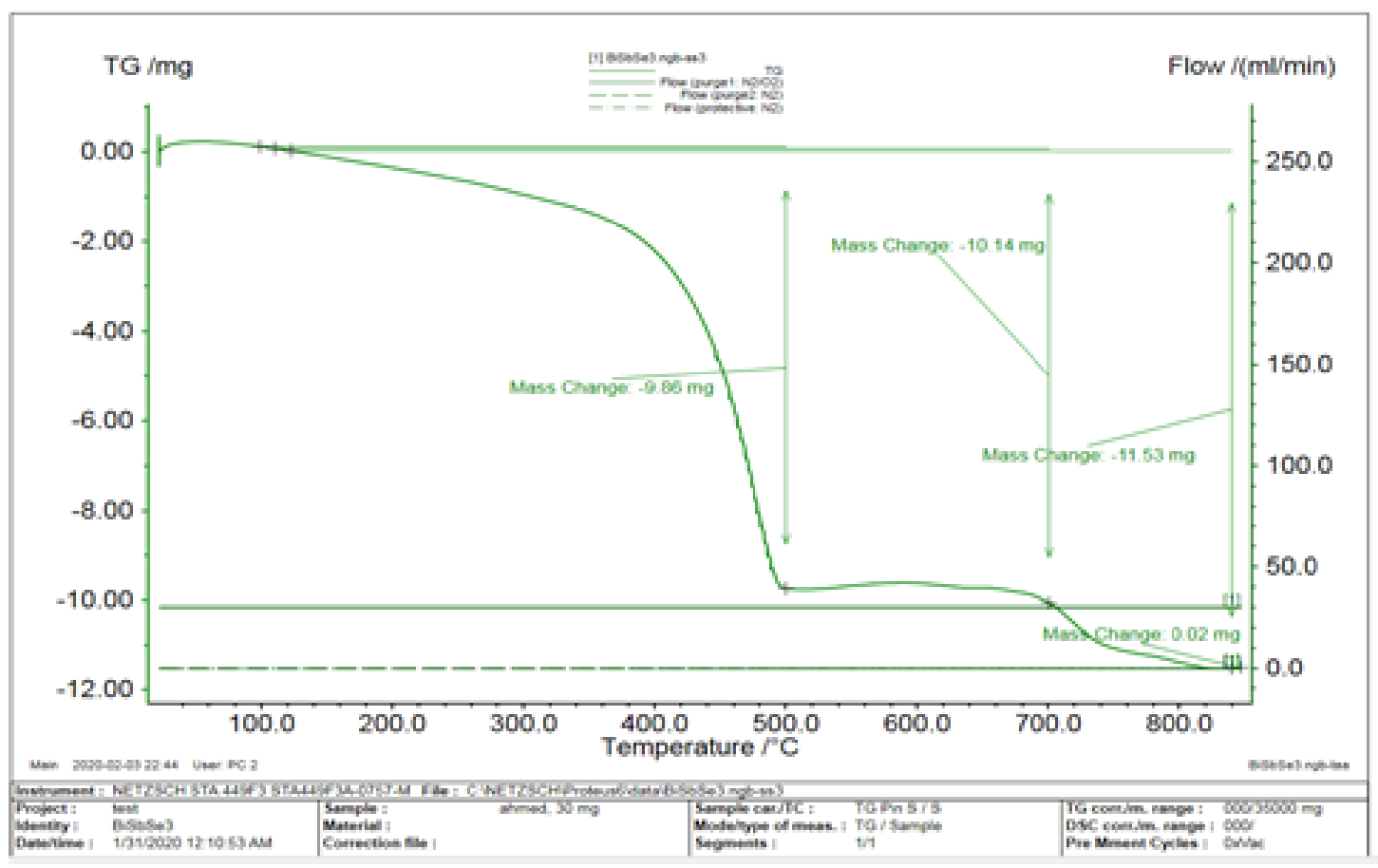

Puc.1. Дериватограмма BiSbSe3

Из рисунка видно что, нагревание образцов проводится в пределах температур $20-850{ }^{0} \mathrm{C}$. Скорость подачи воздуха была 30 мл/мин. Потеря массы при температуре $20-800^{\circ} \mathrm{C}$ составляет 11.53 мг. Для анализа взят образец весом 30 мг. В образце теоретичеки содержится 11.85 мг селен. Потеря массы (в опыте 11.53 мг) произошла за счет селена, a это число согласуется с теоретически вычисленными количествами. Все это показывает правильность формулу соединение $\mathrm{BiSbSe}_{3}$.

Термограммы ДТА показывают, что в кривых $\mathrm{BiSbSe}_{3}$ наблюдались один эндотермический пик (рис.2).

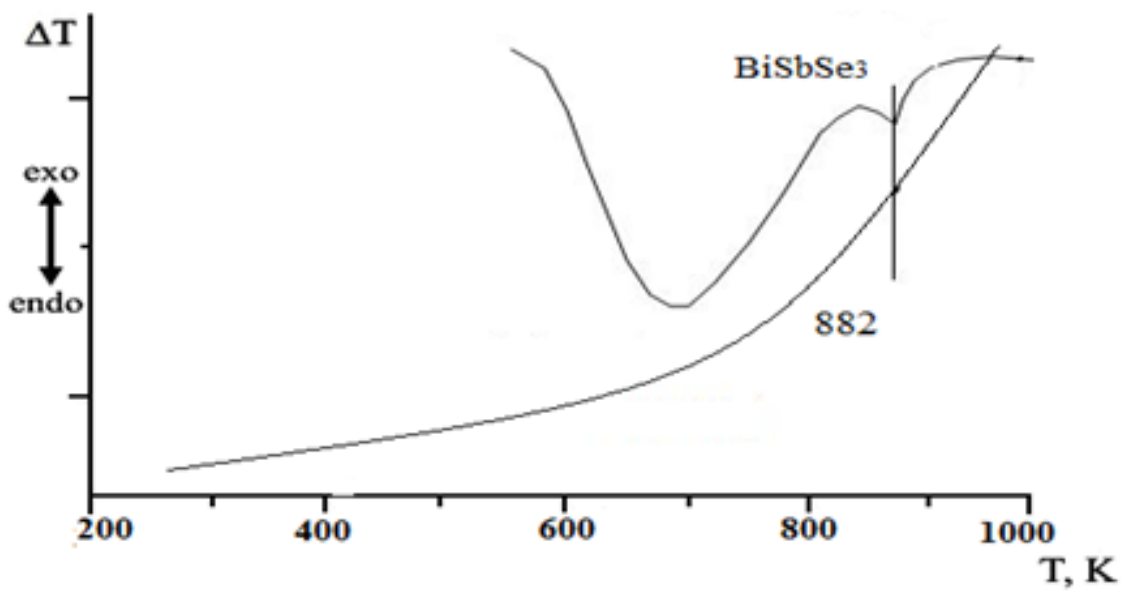

Puc.2. Кривые ДТА BiSbSe3

Температура плавления $\mathrm{BiSbSe}_{3}$ составляет при $\mathrm{Tg}=882 \mathrm{~K}$. При исследовании дифференциально-термическим анализом было установлено, что образца плавятся конгруэнтно.
Рентгенофазовый анализ соединения $\mathrm{BiSbSe}_{3}$ изучен с "D8 ADVANCE" (CuK $\alpha, \lambda=1.5406 \AA ̊$, $0<2 \theta<80^{\circ}$ ), (рис. 3). 


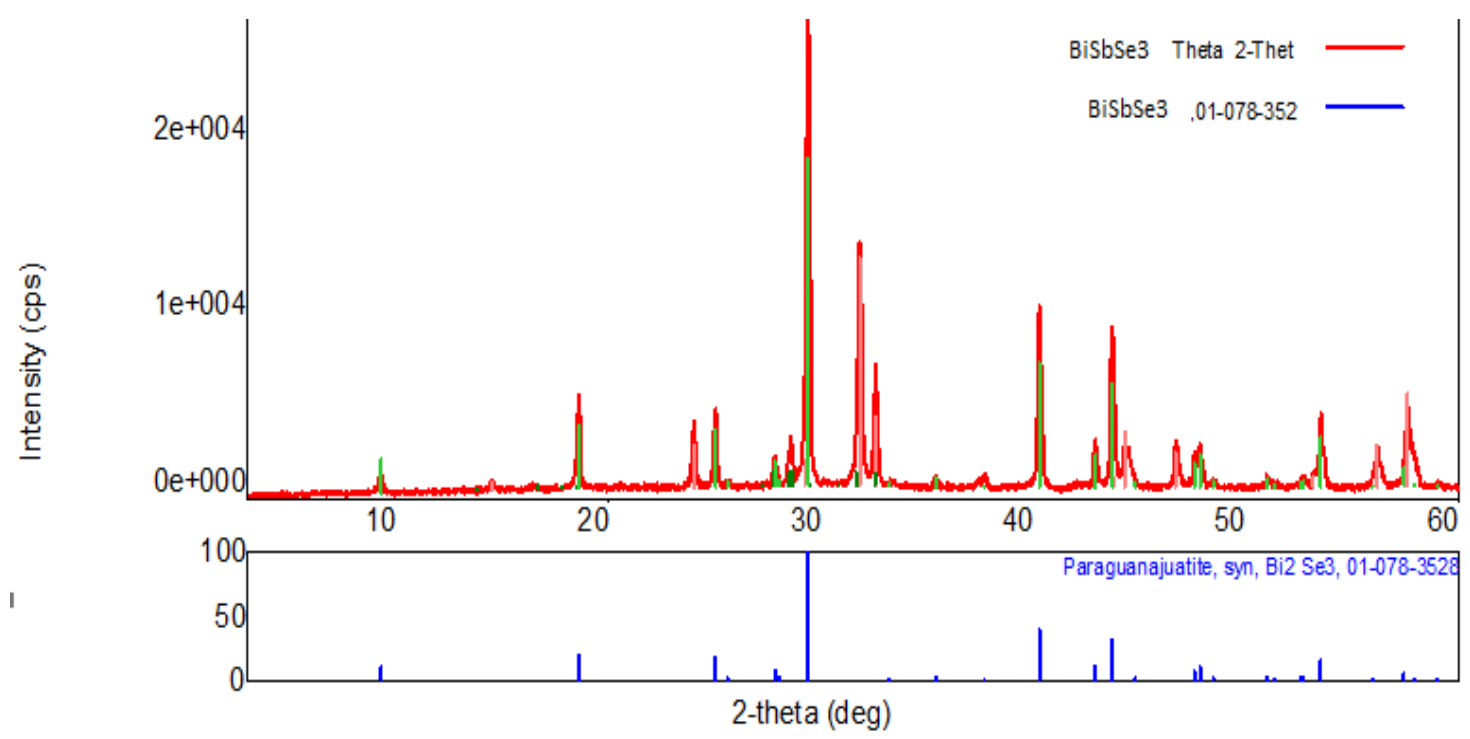

Рис.3. Дифрактограмма соединений BiSbSe3

В рентгенограмме висмут сурьмы селенида интенсивность и положение проявляющих пиков полностью соответствуют стандартам (PDF 01-078 - 352).
Состав соединения висмут сурьма селенида так же анализирован химическими методами, результаты анализов приведены в таблице 1.

Таблица 1

Химический анализ образца

\begin{tabular}{|c|c|c|c|c|c|c|c|}
\hline \multirow{3}{*}{$\begin{array}{c}\text { Тем-ра } \\
\text { получения } \\
\mathrm{BiSbSe}_{3}, \mathrm{~K}\end{array}$} & \multirow{3}{*}{ Образцы, г } & \multicolumn{6}{|c|}{ Компоненты, \% } \\
\hline & & \multicolumn{2}{|c|}{$\overline{\mathrm{Bi}}$} & \multicolumn{2}{|c|}{$\mathrm{Sb}$} & \multicolumn{2}{|c|}{$\mathrm{Se}$} \\
\hline & & теop. & прак. & теop. & прак. & теор. & прак. \\
\hline 453 & 0.5956 & 35.09 & 33.22 & 20.42 & 21.62 & 39.79 & 3716 \\
\hline
\end{tabular}

Результаты анализа показывают, что висмут сурьмы селенида соответствуют формулам $\mathrm{BiSbSe}_{3}$

Изучались влияние температуры на получение, вырастание и формирование нано и

\begin{tabular}{lcrr}
$\begin{array}{l}\text { микрочастиц } \\
\text { солвотермальным }\end{array}$ & $\mathrm{BiSbSe}_{3}$ & \multicolumn{2}{c}{ синтезируемых } \\
изображения этих & частиц & на & электролись \\
эленом
\end{tabular}
микроскопе (рис. 4).

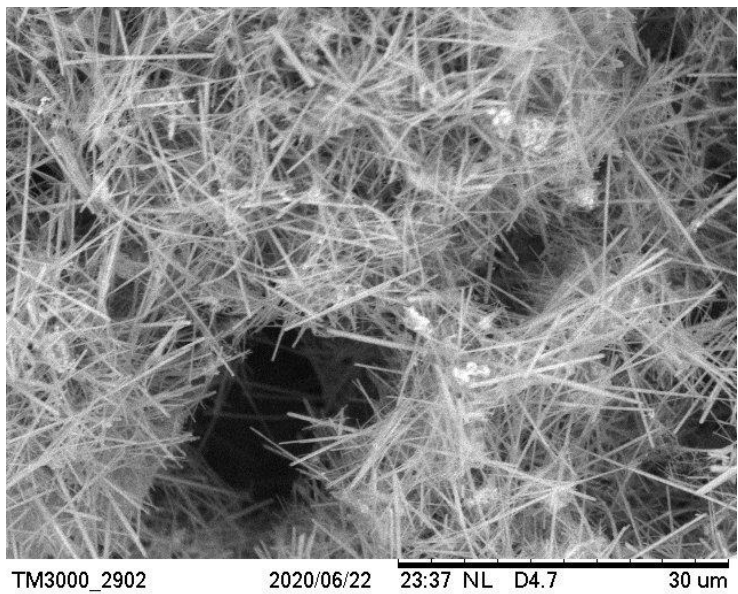

a $б$

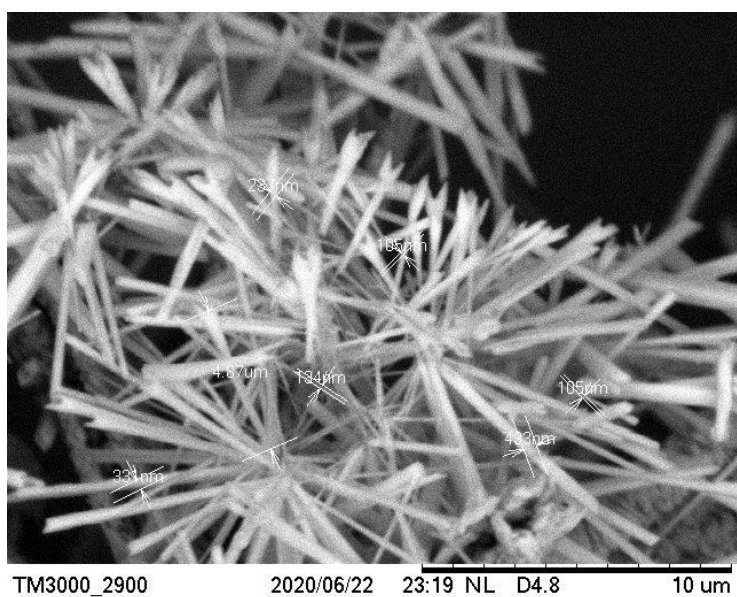

$2020 / 06 / 22 \quad 23: 19 \mathrm{NL} \quad \mathrm{D} 4.8$

Puc. 4. Нано и микрочастицы BiSbSe3 полученные при температуре $453 \mathrm{~K}$ в течение 15 часов: а) увеличение $30 \mu$, б) увеличение $10 \mu м$.

Из рис. 4 видно, что полученные при температуре $453 \mathrm{~K}$ образуются нанопалочки разными размерами и она меняются в интервале, ширина 105-450 $\mathrm{nm}$ и длина меняется соответственно 2-7 $\mu$ м. Мы считаем, что образование и формирование нано и микрочастиц гидротермальным методом зависит от температуры, от времени, а также от жидкой фазы.

\section{ЗАКЛЮЧЕНИЕ}

В первые тройные халькогенидные соединения висмут сурьмы селенида были синтезированы из оксида висмута(III), оксида сурьмы(III), элементарный селена (аморф) и 
гидразин моногидрата в растворе этиленгликолях. Эти соединения (наночастицы) были получены в при температуре 453-463 К в течение 15 часов (pH $=10$-11). Был определен фактический химический состав этих соединений.

\section{СПИСОК ЛИТЕРАТУРЫ}

1.A.H., Farid A.M., Farag A.M., and s. Structural, absorption, dispersion and photo-induced characteristics of thermally vacuum-evaporated $\mathrm{BiSbSe}_{3}$ thin films. Journal of Non-Crystalline Solids, 2015, Volume 416, Pages 50-57

2.Ammar A. H., Abo Ghazala M. S., Alla Farag. Influence of composition on structural, electrical and optical characterizations of Bi48-x Sb x Se52 ternary chalcogenide system. Indian Journal of Physics, 2013, 87(12) DOI: 10.1007/s12648-013-0352-y
3.Navrátil J., Lošták P., Drasar Č., Optical properties of $\left(\mathrm{Bi}_{1-x} \mathrm{Sb}_{x}\right)_{2} \mathrm{Se}_{3}$ single crystals. Bazik solid state physics, 1996, https://doi.org/10. 1002/pssb. 2221940233

4.Xiaoying Liu, Dongyang Wang, Haijun Wu, Jinfeng Wang and s. Intrinsically Low Thermal Conductivity in $\mathrm{BiSbSe}_{3}$ : A Promising Thermoelectric Material with Multiple Conduction Bands. Advansed functional materials, 2018 https://doi.org/10.1002/adfm.201806558

5.Zhang, D., Lei, J., Guan, W., Ma, Z., Cheng, Z., Zhang, L., Wang, C. \& Wang, Y. Cation-anion codoping to enhance thermoelectric performance of $\mathrm{BiSbSe}_{3}$. Materials Science in Semiconductor Processing, 2019. 93, pp 299-303. 